\title{
Multiresolution Denoising for Optical Coherence Tomography: A Review and Evaluation
}

\author{
Aleksandra Pižurica ${ }^{1}$, Ljubomir Jovanov ${ }^{1}$, Bruno Huysmans ${ }^{1}$, \\ Vladimir Zlokolica ${ }^{2}$, Paul De Keyser ${ }^{3}$, Frans Dhaenens ${ }^{3}$ and Wilfried Philips ${ }^{1}$ \\ ${ }^{I}$ Department for Telecommunications and Information Processing (TELIN), Ghent University, Sint-Pietersnieuwstraat 41 , B-9000 \\ Gent, Belgium. \\ ${ }^{2}$ Department for Computer Engineering, Faculty of Technical Sciences, University of Novi Sad, Trg Dositeja Obradovića 6, \\ 21000 Novi Sad, Serbia. \\ ${ }^{3}$ AGFA Healthcare, Septestraat 27, B-2640, Mortsel, Belgium.
}

\begin{abstract}
Recently emerging non-invasive imaging modality - optical coherence tomography (OCT) - is becoming an increasingly important diagnostic tool in various medical applications. One of its main limitations is the presence of speckle noise which obscures small and low-intensity features. The use of multiresolution techniques has been recently reported by several authors with promising results. These approaches take into account the signal and noise properties in different ways. Approaches that take into account the global orientation properties of OCT images apply accordingly different level of smoothing in different orientation subbands. Other approaches take into account local signal and noise covariance's.

So far it was unclear how these different approaches compare to each other and to the best available single-resolution despeckling techniques. The clinical relevance of the denoising results also remains to be determined. In this paper we review systematically recent multiresolution OCT speckle filters and we report the results of a comparative experimental study.

We use 15 different OCT images extracted from five different three-dimensional volumes, and we also generate a software phantom with real OCT noise. These test images are processed with different filters and the results are evaluated both visually and in terms of different performance measures. The results indicate significant differences in the performance of the analyzed methods. Wavelet techniques perform much better than the single resolution ones and some of the wavelet methods improve remarkably the quality of OCT images.
\end{abstract}

Keywords: Optical coherence tomography, image denoising, wavelets

\section{INTRODUCTION}

Optical coherence tomography (OCT) [1]-[5] emerges as a new and promising non-invasive medical imaging modality. Next to its primary application in ophthalmology, OCT is becoming increasingly popular diagnostic tool in many other medical fields, including dermatology, gastroenterology, dentistry and endoscopic intra-arterial imaging. The main advantages of OCT are a remarkably high resolution both in depth and transversally, as well as non-invasive, and contact-free imaging. A disadvantage is a limited penetration depth in scattering media.
Likewise all coherent imaging systems, OCT suffers from speckle noise. Appearing as a random granular pattern, speckle obscures small and low-intensity features. While sharing some common properties with other types of speckle noise (like, e.g., in ultrasound images) OCT speckle has some characteristic properties and so does the OCT signal itself. A well-designed OCT despeckling method will benefit from taking into account these characteristic signal and speckle properties.

The use of multiresolution (wavelet-based) techniques has been recently reported by several authors with promising results. These approaches take into account the signal and noise properties in different ways. Approaches that take into account the global orientation properties of 
OCT images apply accordingly different level of smoothing in different orientation subbands. Other approaches take into account local signal and noise covariance's. So far it remains unclear how these different approaches compare to each other and to the best available single-resolution despeckling techniques. The clinical relevance of the denoising results also remains to be determined. In this paper, we review systematically recent multiresolution OCT speckle filters, we report the results of a comparative experimental study, and we give a critical discussion of our findings. We evaluate the techniques both on phantom images and on real OCT images and on a software phantom image.

This paper is organized as follows. Section II reviews briefly main concepts of OCT imaging: the formation of OCT images (Section II-A), some applications (Section II-B) and noise properties (Section II-C). A systematic classification of representative noise reduction methods for OCT is in Section III. Section IV starts with a brief overview of the wavelet transform and wavelet denoising principles and then describes four representative approaches for OCT speckle reduction in the wavelet domain. The performance evaluation is in Section V. We divide this performance evaluation into visual assessment (Section V-A) and objective performance evaluation (Section V-B). Next to the widely used performance measures, we adapt some other measures for making them applicable to our problem and we also define a new measure for texture preservation. A discussion is in Section VI and the conclusions in Section VII.

\section{COHERENCE TOMOGRAPHY IMAGING}

\section{A. Formation of OCT images}

Optical Coherence Tomography (OCT) [1], [3]-[5] can be briefly described as echography with light. Due to the speed of light however, practical OCT systems are not time-of-flight based (while ultrasound is). Optical interferometry is used as an indirect way to visualize coherently reflected or scattered light. In the simplest form, a partially coherent light source is illuminating a Michelson interferometer (see Fig. 1). In the reference arm, a mirror is moved (speed in the $\mathrm{kHz}$ range, e.g. with a voice coil) so that constructive interference can only happen at a depth in the tissue corresponding with the position of the reference mirror at each moment (taking into account the ratio of the refractive indices in the tissue and in the air). This way, a vertical linear image ("A-Scan" or "needle image") of the tissue is produced. By scanning the beam transversally, a 2D cross-section image of the tissue can be made. If the whole construction is additionally moved in a direction perpendicular to the transversal direction, a full 3D image volume can be imaged.
The light source needs to be partially coherent, in order to allow constructive interference only at a certain depth. With a coherent source (laser), a constructive interference could arise from wherever in the tissue (e.g., after multiple scattering) and there would not be any depth ( $z$-axis) resolution. In fact, one can prove that the $z$ axis resolution of an OCT system is proportional to the bandwidth of the light source, thus it is crucial to use sources with a bandwidth as high as possible, yet with limited cost. Currently, SLDs (Super Luminescent Diodes) fit this purpose best with bandwidths of 50-100 $\mathrm{nm}$ for a $1300 \mathrm{~nm}$ wavelength.

A nice feature of OCT is that this z-resolution is fully decoupled from the resolution in the perpendicular directions (i.e., from $x$ - and $y$-resolution), which is determined by the optics of the system. This is not the case, e.g., with confocal microscopy. The choice of wavelength is a compromise between better resolution at smaller wavelengths versus less scatter at higher wavelengths.

The contrast or signal is based on Fresnel reflection: only where there are significant changes of the index of refraction (within the coherence length) a signal will be generated. It can be shown that the detector signal is proportional to the square root of the sample power reflectivity. The remarkable result is that relative contrast changes as small as $10^{-6}$ can still be detected since they are "square rooted" to a $1 / 1000$ contrast, which can still be detected, even with a normal CCD. In other words: the small contrast variation is amplified considerably by the interferometer mechanism.

A typical waveform coming from a needle scan is shown in Fig. 2 - the "bumps" indicate depths where there was a significant change in tissue characteristics (i.e., in the refractive index). This signal is then typically demodulated by first of all rectifying, and then filtering with some kind of low-pass or band pass filter to detect the actual reflections. Fig. 3 illustrates the resulting, demodulated waveform.

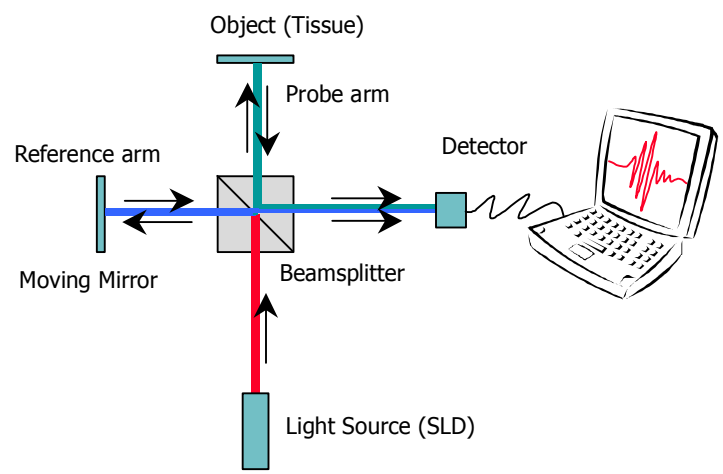

Fig. 1. Principle of an OCT interferometer. 


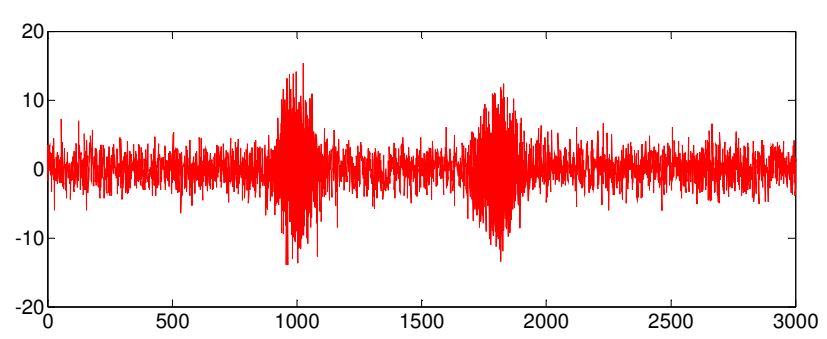

Fig. 2. Raw A-Scan OCT signal acquired by probing vertically down into an object.

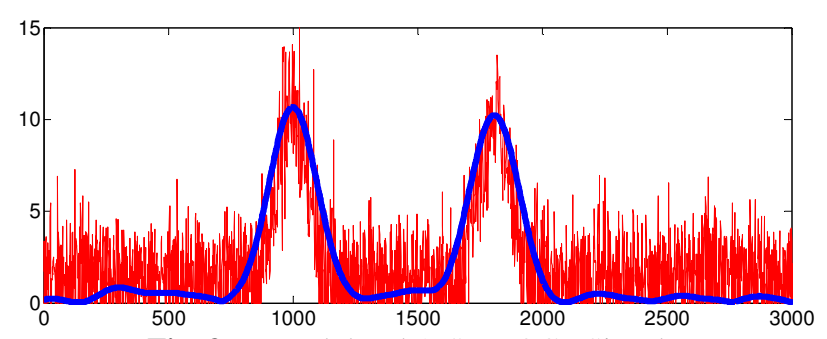

Fig. 3. Demodulated A-Scan OCT Signal.

Assigning the measured intensity values in a pixel matrix results in an image representative of the tissue structure changes.

\section{B. Some applications of OCT}

The very first commercialized applications are in the field of opthalmology: OCT enables a totally noninvasive view of the retina tissue structure. This allows to precisely diagnose and monitor diseases like Age-related Macular degeneration (AMD), retinopathy, etc. - albeit that most methods still have relatively low resolution (10 microns) and do not cope well with eye movements.

The capability of bringing microscopic detail in vivo equals a kind of "virtual biopsy" which potentially could replace many invasive biopsy procedures to support diagnosis - in many fields beyond ophthalmology. The in vivo capability, combined with the totally harmless character of the near-infrared radiation, will also allow quality control (e.g. verification of cancer-free tissue after surgery, verification of lens thickness during LASIK procedures etc.). The weakness of the limited working depth (in practice in the order of $1 \mathrm{~mm}$ currently) obviously has to be overcome by miniaturizing the technology so that it could be used in endoscopes and even catheters. A thorough review of OCT applications is in [5].

\section{Speckle noise in OCT}

OCT images, as well as all other imaging modalities that involve a coherent light source, are affected by speckle noise. Speckle, arising from constructive and destructive interferences of the backscattered waves appears as a random granular pattern [6] that significantly degrades image quality and complicates further image processing tasks, like image segmentation and edge detection. In addition to the optical properties (like multiple scattering and phase aberrations of the propagating light beam) and target motion, the speckle formation is also influenced by the physical parameters of the imaging device: size and temporal coherence of the light source and the aperture of the detector [4].

Speckle is well modeled by a multiplicative noise. In denoising studies, it is often simplified that a logarithmical transform converts multiplicative speckle noise into additive white Gaussian noise [7]-[9]. In [10], it is shown that this assumption is oversimplified and a preprocessing procedure is proposed, which modifies the acquired images so that the noise in the logtransformation domain becomes close to white Gaussian noise.

The marginal statistics of (fully developed) speckle is well modeled by a Rayleigh distribution [11], [12]. The Rayleigh model proves to be a good model for the firstorder statistics of OCT images as well [13], [14], even though under certain conditions a Gaussian model holds [15]. An analytical model that describes the performance of OCT signals in both single and multiple scattering regimes is presented in [16] and [17] and the influence of multiple scattering on the measurement of the total attenuation coefficient is described in [18] and [19]. In [20], the correlation between OCT speckle and the statistical and optical properties of the sample tissue are discussed. Higher order speckle statistics (speckle autocorrelation, roughness and randomness of the structure, etc.) are studied, e.g., in [12], [21]-[23] and in classical optical literature [24].

\section{SPECKLE REDUCTION IN OCT}

Standard despeckling methods include the Lee [25], Kuan [26] and Frost [27] filters, which use the second order statistics within a minimum mean squared error estimation approach and a multiplicative speckle model. A comparative analysis of these and related filters is, e.g., in [28]. For OCT images, the Rotating Kernel Transform (RKT) filter [29], [30] is one of the best performing single-resolution filters. The RKT technique filters an image with a set of oriented templates (kernels) and keeps the largest filter output at each pixel [29]. The filtering kernel consists of zeroes and ones and is typically an elongated, line-like structure that is rotated in small discrete steps through $360^{\circ}$. In essence, the RKT filter calculates the weighted average of the pixel values in an elongated neighborhood, repeats that along different directions and retains the maximum result. This technique is efficient, but rather ad hoc and uses no information concerning the speckle statistics. Several multi-resolution (wavelet-based) speckle filters will be reviewed in the next Section. 
Noise reduction can be applied before or after OCT image formation. The techniques that are applied to the magnitude of the OCT interference signal (i.e., after an OCT image is formed) are also called image post processing techniques. Other techniques are applied to the complex interference signal itself (complex domain methods) [4], [31].[35]. In this paper, we address the first category.

We can make a distinction between generic techniques, developed for other (or for more general) imaging modalities, which can be applied to OCT (like various speckle filters [36]-[41] or filters for correlated noise [42]) and techniques specifically developed or adapted for OCT [29], [30], [43], [44].

Another possible categorization is into single-resolution and multi-resolution techniques, such as wavelet-based techniques [39]-[44].

Some methods suppress only noise, while others attempt at deblurring as well and apply deconvolution combined with noise suppression. The deconvolution methods developed for OCT require a priori information about the point-spread function of the imaging optics, as well as the optical properties of the imaged tissue.

In Table 1, we give an overview of some representative OCT speckle reduction techniques and we classify them according to the criteria mentioned above.

\begin{tabular}{|l|c|c|c|c|}
\hline \multicolumn{1}{|c|}{ Method } & PP or C & G or O & N or D & I or T \\
\hline$[31]$ & $\mathrm{C}$ & $\mathrm{O}$ & $\mathrm{N}$ & $* * *$ \\
\hline$[32],[34],[45]$ & $\mathrm{C}$ & $\mathrm{O}$ & $\mathrm{D}$ & $* * *$ \\
\hline$[29]$ & $\mathrm{PP}$ & $\mathrm{O}$ & $\mathrm{N}$ & $\mathrm{I}$ \\
\hline$[36]-[38],[46]$ & $\mathrm{PP}$ & $\mathrm{G}$ & $\mathrm{N}$ & $\mathrm{I}$ \\
\hline$[43],[44]$ & $\mathrm{PP}$ & $\mathrm{O}$ & $\mathrm{N}$ & $\mathrm{T}$ \\
\hline$[39]-[41],[47],[89]$ & $\mathrm{PP}$ & $\mathrm{G}$ & $\mathrm{N}$ & $\mathrm{T}$ \\
\hline
\end{tabular}

Table 1. Classification of some state of the art OCT speckle reduction techniques. From left to right: image Post Processing (PP) or Complex domain processing (C); Generic technique (G) or adapted to OCT $(\mathrm{O})$; only Noise suppression $(\mathrm{N})$ or also Deconvolution (D); working in the Image domain (I) or working in a Transform domain (T) (if relevant).

\section{WAVELET BASED OCT FILTERS}

In this Section, we review several representative wavelet based denoising methods for OCT ranging from thresholding to vector based minimum mean squared error estimation. Other notable examples of wavelet based OCT denoisers include the optimal non-linear wavelet thresholding (ONWT) method of [43], originally developed for ultrasound [48], which applies softthresholding, with image dependent and sub-band dependent thresholds and the multi-dimensional method of [49], which processes multiple OCT image slices by making use of both spatial and temporal correlations.

\section{A. Wavelet transform}

The wavelet transform reorganizes image content into a low-resolution approximation and a set of details of different orientations and different resolution scales. A fast algorithm for the discrete wavelet transform is an iterative filter bank algorithm of Mallat [50], where a pair of high-pass and low-pass filters followed by down sampling by two is iterated on the low-pass output. In a non-decimated wavelet transform that we consider in this paper, down sampling is excluded, and instead the filters are up sampled at each decomposition stage as explained later in the text. The outputs of the low pass filter are the scaling coefficients and the outputs of the high-pass filter are the wavelet coefficients. At each decomposition level, the filter bank is applied sequentially to the rows and to the columns of the image. Low-pass filtering of both the rows and the columns yields the low-pass LL sub-band and other combinations of low-pass and high-pass filtering yield the wavelet sub-bands at different orientations: High-pass filtering of rows and low-pass filtering of columns (HL) yields horizontal edges and the opposite combination (LH) yields vertical edges, while high-pass filtering of both the rows and the columns $(\mathrm{HH})$ yields highest frequency information, corners and edges that are close to diagonal orientations. The $j$-th decomposition level yields the coefficients at the resolution scale $2^{j}$.

Critically sampled (orthogonal) wavelet transform is not shift-invariant. In such a representation, small errors in estimation of the coefficients result in annoying blobs and ringing artifacts. Denoising performance is much improved when using redundant and (nearly) shiftinvariant transforms. The resulting gain in PSNR is often $1 \mathrm{~dB}$ or even more [52]. Common approaches include using non-decimated wavelet transform [52,53,74-77], dual-tree complex wavelet transform [86] and cyclespinning [51]. Cycle-spinning consists of denoising multiple cyclical shifts of the image and averaging over unshifted results, which yields a similar improvement over the critically-sampled case as the non-decimated transform [52]. More recent approaches achieve further improvements in the denoising performance by using highly redundant representations with multiple orientation bands such as curvelets $[87,88]$ and steerable pyramids $[42,47,89]$.

We use a non-decimated wavelet transform implemented with the algorithm à trous [53]. The algorithm inserts $2^{j}-1$ zeroes (i.e., holes, French trous) between the filter coefficients at the resolution level $j$. The size of each wavelet sub-band equals the size of the input image. For compactness, denote the spatial position vector $[m, n]$ by a single index $l$, and denote the scaling coefficients at the resolution level $j$ by $a_{l}^{j}$ and the wavelet coefficients 
at the corresponding scale in three orientation sub-bands by $y_{l}^{j, H L}, y_{l}^{j, L H}$ and $y_{l}^{j, H H}$. With this notation, we have:

$$
\begin{aligned}
& a_{l}^{j+1}=\left(\bar{h}^{j} \bar{h}^{j} * a^{j}\right)_{l} \\
& y_{l}^{j+1, H L}=\left(\bar{g}^{j} \bar{h}^{j} * a^{j}\right)_{l} \\
& y_{l}^{j+1, L H}=\left(\bar{h}^{j} \bar{g}^{j} * a^{j}\right)_{l} \\
& y_{l}^{j+1, H H}=\left(\bar{g}^{j} \bar{g}^{j} * a^{j}\right)_{l}
\end{aligned}
$$

Decomposing an image into $J$ decomposition levels yields a wavelet image representation consisting of $3 J+$ 1 subbands $\left[a^{J},\left\{\mathbf{x}^{j, H L}, \mathbf{x}^{j, L H}, \mathbf{x}^{j, H H}\right\}_{1 \leq j \leq J}\right]$, i.e.,

$$
\begin{aligned}
& a_{l}^{0}=\left(h^{J} h^{J} * a^{J+1}\right)_{l}+ \\
& \sum_{j=1}^{J}\left(\left(g^{j} h^{j} * y^{j+1, H L}\right)_{l}+\left(h^{j} g^{j} * y^{j+1, L H}\right)_{l}+\left(g^{j} g^{j} * y^{j+1, H H}\right)_{l}\right)
\end{aligned}
$$

where the input image is approximated by $\mathbf{a}^{0}$ with a negligible error [53].

\section{B. Preliminaries and Notation}

Wavelet transform is attractive for denoising since it compresses the essential information about image/signal into a few large coefficients, while input noise tends to be spread among all the coefficients. Hence even in the presence of large amounts of noise, the wavelet coefficients representing strong image edges can be identified. This facilitates construction of discontinuityadaptive filtering, where noise is smoothed out without excessive blurring of significant image edges and other important image details.

A common shrinkage approach is thresholding [54]-[57], where the coefficients with magnitudes below a certain threshold are treated as "non significant" and are set to zero, while the remaining, "significant" ones are kept unmodified (hard-thresholding) or reduced in magnitude (soft-thresholding). Shrinkage estimators can also result from a Bayesian approach [41], [42], [52], [58]-[76], which imposes a prior distribution on noise-free data. Inter- and intrascale dependencies among the wavelet coefficients are often accounted for by using local contextual measurements (such as locally averaged magnitudes, local variances, and related local spatial activity indicators) [42], [52], [70], [77] or more complex contextual models, such as Hidden Markov Tree (HMT) models [68], [69], [78] and Markov Random Field (MRF) models [74]-[76], [79]. Other recent approaches use spatial connectivity information to refine the selection of the wavelet coefficients in a hardthresholding procedure (so-called feature-based or "geometric" hard-thresholding [83]) explained in Section IV.D.
In the following, we will assume that a logarithmic transform has been applied to the input image, which transforms the input multiplicative noise into additive Gaussian noise. Due to linearity of the wavelet transform, the noise in the wavelet domain remains additive Gaussian. We use the following notation

$$
w_{l}^{j, d}=y_{l}^{j, d}+n_{l}^{j, d}
$$

where $y_{l}^{j, d}$ denotes the noise-free coefficient component at spatial position $l$, resolution level $j$ and orientation $d$. $n_{l}^{j, d}$ is a Gaussian distributed noise contribution and $w_{l}^{j, d}$ the observed noisy coefficient.

\section{Spatially adaptive thresholding for OCT}

Effective and simple denoising approach is wavelet softthresholding [54] with a level-dependent threshold $T$, where $\hat{y}_{l}^{j, d}=\operatorname{sgn}\left(w_{l}^{j, d}\right) \max \left(\left|w_{l}^{j, d}\right|-T, 0\right)$. The choice of the threshold $T$ has been a subject of many research papers. A well known approach is the so-called universal threshold [54]: $T=\sigma_{n} \sqrt{2 \log N}$, where $\sigma_{n}$ is the noise standard deviation and $N$ is the number of the coefficients in a given subband. The rationale behind this threshold is to remove all the coefficients that are smaller than the expected maximum of i.i.d. normal noise. Many other threshold selection procedures exist, see, e.g., [90]. Despite its theoretically proved optimality for a broad class of functions [54], [55], thresholding with spatially fixed threshold tends to over smooth the image. Highquality denoising is achieved by making the threshold dependent on the sub-band statistics but also on the local spatial context.

An efficient method for the suppression of additive white Gaussian noise employing sub-band adaptive and spatially adaptive threshold $T_{l}^{j, d}$ was proposed by Chang et al in [52], where it was called spatially adaptive wavelet thresholding (SAWT). The SAWT estimator is

$$
\hat{y}_{l}^{j, d}=\operatorname{sgn}\left(w_{l}^{j, d}\right) \max \left(\left|w_{l}^{j, d}\right|-T_{l}^{j, d}, 0\right)
$$

with

$$
T_{l, j}^{d}=\frac{\sigma_{n}^{2}}{\left(\sigma_{y}^{2}\right)_{l}^{j, d}}
$$

where $\sigma_{n}^{2}$ is the noise variance (equal in all the subbands when orthogonal wavelets are used) and $\left(\sigma_{y}^{2}\right)_{l}^{j, d}$ is the signal variance, estimated from the coefficients with similar spatial context within the same subband. 
A modification of this filter that takes into account specific properties of OCT images was proposed by Adler, Ko and Fujimoto in [44]. There, SAWT is applied to the logarithmically transformed image (which maps multiplicative type of noise into additive) and with an increased threshold in the vertical subbands. The subsequent exponential operation yields the denoised OCT image. The modified thresholds account for the OCT statistics as follows. The actual signal in OCT images consists mainly of horizontal edges arising from reflections at the layer boundaries. Hence most of the edge information is contained within LH subbands and some of it in $\mathrm{HH}$ subbands, while vertical HL subbands contain mainly noise. Therefore the authors reason that increasing the threshold in HL subbands by a constant multiplier $K$ decreases further noise with a minimal effect on the edge sharpness. In experiments they find that the SNR is maximized for $K=4$. We will use this filter in our evaluation, where we shall denote it by OCTWT (from OCT-specific wavelet thresholding).

\section{Feature based wavelet thresholding}

In classical hard thresholding [55], the coefficients below a certain threshold are zeroed while the remaining ones are kept unchanged. This procedure introduces less smoothing than the soft-thresholding but leaves more annoying blob-like artifacts. However the idea of keeping a subset of the information-carrying coefficients (selected according to well-defined inter- and intrascale criteria) and zeroing all the others has led to many efficient denoising algorithms [80], [81] reflecting early Marr's vision of image reconstruction from its edges [82]. Also, in medical image processing some researchers advocate keeping unchanged the coefficients that are identified as significant in order to avoid blurring of diagnostically relevant features. This should be approached critically because at positions where the coefficients are wrongly selected as significant more visible artifacts are likely to arise.

A recent method of Balster et al [83] reconstructs the denoised image from a selected subset of the wavelet coefficients, where the selection criterion combines the coefficient magnitude, spatial regularity and regularity across the scales. The spatial regularity criterion means that only coefficients that form connected edge-like structures are candidates for the selection. The regularity across the scales refers to the evolution of the coefficient magnitude from fine to coarse scales. This criterion, using the same reasoning as [80], [81] discards the coefficients that diminish quickly with the increasing the scale, because these are likely to represent noise.

In a first stage, the method of [83] selects the "valid" coefficients according to the magnitude criterion. This results in a preliminary binary classification

$$
I_{l}^{j, d}=\left\{\begin{array}{l}
1, \text { if } \mid w_{l}^{j, d} \triangleright \tau \\
0, \text { else }
\end{array}\right.
$$

where $\tau$ is a threshold proportional to the noise standard deviation. In the next stage, the method examines spatial regularity by counting the number of spatially connected labels " 1 " around each nonzero label $I_{l}^{j, d}$. The number of connected labels " 1 " defines the support value $S_{l}^{j, d}$ for the corresponding spatial position (and for the other spatial positions with label "1" spatially connected to it) ${ }^{1}$. The preliminary binary classification is refined based on the intra-scale support value $S_{l}^{j, d}$ and based on a recursive inter-scale criterion:

$$
J_{l}^{j, d}=\left\{\begin{array}{l}
1, \text { if } S_{l}^{j, d}>s \text { or } J_{l}^{j+1, d} I_{l}^{j, d}=1 \\
0, \text { else }
\end{array}\right.
$$

According to this criterion, the coefficient $w_{l}^{j, d}$ is selected as significant if it is locally supported $\left(S_{l}^{j, d}>s\right)$ or if it is large $\left(I_{l}^{j, d}=1\right)$ and its parent is significant $\left(J_{l}^{j+1, d}=1\right)$. The denoised image is then reconstructed from the selected subset of the coefficients. In our notation:

$$
\hat{y}_{l}^{j, d}=J_{l}^{j, d} w_{l}^{j, d}
$$

In our study, we apply this method to the logarithmically transformed OCT image and we denote it as FBT (from feature-based thresholding). A related method with an extension to soft-thresholding was proposed in [84].

\section{E. Spatially adaptive shrinkage with Gamma- Exp model}

The adaptive speckle suppression scheme from [40] estimates the noise-free coefficients as

$$
\hat{y}_{l}^{j, d}=q_{l}^{j, d} w_{l}^{j, d}
$$

where $q_{l}^{j, d}$ is a shrinkage factor $0 \leq q_{l}^{j, d} \leq 1$, which shrinks wavelet coefficients depending on the estimated probability that the coefficient represents an important noise-free image feature. The corresponding probability is estimated based on two sources of information: (i) coefficient magnitude and (ii) spatial context information.

Let us denote the coefficient magnitude by $m_{l}^{j, d}$, and let us associate with each wavelet band $\mathbf{w}^{j, d}$ a mask $\mathbf{x}^{j, d}$ of

\footnotetext{
${ }^{1}$ All the spatially connected positions with label " 1 " have the same support value.
} 
binary labels, where $x_{l}^{j, d}=0$ ("non-edge" label) if $w_{l}^{j, d}$ represents mainly noise, and $x_{l}^{j, d}=1$ ("edge" label) if $w_{l}^{j, d}$ represents useful signal. In the following, for compactness, we suppress the indices that denote the scale and orientation, unless in case where explicitly needed. The likelihood of $m_{l}$ given the label value $x_{l}$ will be denoted by $p\left(m_{l} \mid x_{l}\right)$. Further on, the label value $x_{l}$ is a realization of a random variable $X_{l}$. Hence capital letters will denote random variables and the corresponding small letters their realizations.

The method of [40] defines the shrinkage factor as follows

$$
q_{l}=P\left(X_{l}=1 \mid m_{l}, \mathbf{x}_{l}^{\prime}\right)
$$

where $\mathbf{x}_{l}^{\prime}$ denotes the mask labels at all positions except $l$. It is shown in [40] that this expression can be rewritten as

$$
q_{l}=\frac{\xi_{l} \eta_{l}}{1+\xi_{l} \eta_{l}}
$$

where $\xi_{l}$ is the likelihood ratio at the current position $l$

$$
\xi_{l}=\frac{p\left(m_{l} \mid X_{l}=1\right)}{p\left(m_{l} \mid X_{l}=0\right)}
$$

and $\xi_{l}$ is derived from a spatial surrounding $\partial_{l}$ as follows [40]:

$$
\eta_{l}=\exp \left(\gamma \sum_{k \in \partial_{l}}\left(2 x_{k}-1\right)\right)
$$

Note that if there is equal number of "edge" and "nonedge" labels in the neighborhood $\partial_{l}$ then the neighborhood information is "neutral" and (13) yields $\eta_{l}=1$. This means that in case where there is equal number of edge and non-edge labels in $\partial_{l}$ the spatial context information does not influence the calculation of the shrinkage factor in Eq (12). In that case, the suppression factor depends only on the coefficient magnitude (through $\xi_{1}$ ). If the neighborhood $\partial_{l}$ contains a majority of edge (" 1 ") labels then $\eta_{l}$ is greater than 1 and it increases the shrinkage factor in (12). The opposite is true when there is a majority of "non-edge" (zero) labels in the corresponding neighborhood. In this way, the spatial context information, expressed through $\eta_{l}$, favors suppressing less those wavelet coefficients that are surrounded by the majority of "edge" coefficients and it favors suppressing heavier the isolated big coefficients (that are more likely to originate from noise).
The binary labels are estimated using a preliminary coefficient classification as follows:

$$
x_{l}^{j, d}=\left\{\begin{array}{l}
1, \text { if }\left|w_{l}^{j, d} \| \hat{y}_{l}^{j+1, d}\right|>\sigma^{j, d} \\
0, \text { else }
\end{array}\right.
$$

where $\sigma^{j, d}$ is the noise standard deviation in the subband at scale $j$ and orientation $d$. In this process, a coarse-to fine strategy is employed, where a previously denoised wavelet subband from a coarser scale $j+1$ is used to estimate the edge positions at the next finer scale $j$.

For the calculation of the likelihood ratios, the following model was proposed in [40]:

$$
\begin{gathered}
p\left(m_{l} \mid X_{l}=0\right)=\frac{1}{a} \exp \left(-m_{l} / a\right) \\
p\left(m_{l} \mid X_{l}=1\right)=\frac{m}{2 b^{3}} \exp \left(-m_{l} / b\right)
\end{gathered}
$$

where the parameters $a$ and $b$ are computed automatically during the denoising procedure by maximum likelihood approach. Like in the previous two methods, we apply this method to the logarithm of the OCT image. We shall denote this method by WGE (referring to wavelets and to gamma-exponential priors).

\section{F. Denoising methods taking into account noise correlation}

The methods presented in the previous Sections ignore noise correlation. A starting approximation there was that the logarithmic transform maps the OCT speckle into additive white Gaussian noise.

Even though this approximation is for simplicity often employed in speckle denoising literature, there exist more elaborate models that take into account noise correlation. A wavelet domain denoising method of Portilla et al [47] model noise by a correlated Gaussian model and the signal by a correlated Gaussian Scale Mixture (GSM) model. This method is an extension of [42] with the main difference that the noise covariance is estimated directly from the image using a generalized expectation maximization algorithm. The method applies minimum mean squared error estimator on the vectors of coefficients from (overlapping) blocks, resulting in a Wiener-like estimator under the assumed prior model. For details, we refer to [42], [47]. The application of this method to OCT was demonstrated in [47]. A related model with spatially varying GSMs is in [89]. In this study, we use the Bayesian least squares estimator with the GSM model (BLS-GSM) from [47]. The software for this method is publicly available at the web-site http://www.io.csic.es/PagsPers/JPortilla/denoise/software /index.htm. 


\section{PERFORMANCE EVALUATION}

We evaluate the performance of the analyzed speckle filters on OCT images taken from six different three dimensional volumes and on a software phantom image. The scans were made on different biological tissues including liver, finger tip, breast and rat bone, with the following OCT apparatus: SkinDex 300 (ISIS Optronics, Mannheim Germany). The scan depth is $904 \mu \mathrm{m}$ and the width is $1005 \mu \mathrm{m}$ (i.e., approx $1 \mathrm{~mm}$ ). The distance between the slices was $1.1 \mu \mathrm{m}$ and the size of each image slice (in axial $\times$ transverse direction) is $556 \times 500$ pixels. One volume consisted of 60 slices, another of 126 and the remaining three of 252 slices. Out of these volumes, we selected a number of slices for objective evaluation and for visual assessment of the filtering results.

We test two single-resolution filters:

- Lee [25] and RKT [29], [30]

and four multiresolution filters, that we denote as

- OCTWT (Wavelet Thresholding for OCT) [44] (Section IV-C);

- $\quad$ FBT (Feature based Wavelet Thresholding) [83] (Section IV-D);

- WGE (Wavelet shrinkage with GammaExponential model) [40] (Section IV-E);

- BLS-GSM (Bayesian Least Square estimator with Gaussian Scale Mixture prior) [42], [47] (Section IV-F).

First we show visual results and then we define and evaluate some objective performance measures.

\section{A. Visual comparison}

Visual results on two test images from Fig. 4 are shown in Fig. 5, Fig. 6 and Fig. 7. Fig. 5 and Fig. 6 show a comparison between the six tested methods. For the WGE method, the results are illustrated for two different values of the parameter $\gamma$. These results demonstrate that the single resolution filters are inferior with respect to the wavelet based ones. Among the wavelet based methods, the FBT approach achieves a very good contrast and edge preservation, but seems to flatten severely textures. The other three multiresolution methods (OCTWT, WGE and BLS-GSM) are more conservative in this respect and preserve better fine image structures.

We compare visual results of the three best performing methods on enlarged details in Fig. 7. The parameter of the WGE method was in this case set to $\gamma=0.4$, which was visually found as a best compromise between noise suppression and detail preservation. The three results in Fig. 7 are very similar in quality; the WGE method is most conservative of all when it comes to point-like structures. The BLS-GSM and OCTWT methods give a natural appearing mildly smoothed textures. Vague horizontal artifacts are visible in the background region of the OCTWT result, which is apparently due to different suppression factors in differently oriented subbands. The result in Fig. 8 illustrates clearly that noise reduction enhances visually OCT images.

\section{B. Objective performance measures}

To assess objective performance of the analyzed filters, we define several regions of interest (ROI), similarly as it was done in [44]. Fig. 9 shows seven chosen regions of interest being small rectangular areas with interesting details, edges or textural features inside. The largest, elongated rectangle in this image is used only to assess the background statistics. In the same way, we choose the ROI's on other 12 images displayed in Fig. 10.

We use some standard objective performance measures, but we also define new ones because the standard measures have some limitations that we discuss below. Some of these measures are global, i.e., evaluated over the whole image, while others are local, i.e., evaluated over regions of interest. A global performance measure is Signal to Noise Ratio that we evaluate as SNR= $20 \log \left(I_{\max } / \sigma_{b}\right)$, where $I_{\max }$ is the maximum value in the processed image, and $\sigma_{b}$ is the standard deviation of noise in the background region, which estimates the standard deviation of the remaining noise.

From the standard local measures, we use contrast to noise ratio and equivalent number of looks. In addition, we define a local edge-preservation measure and we introduce a new texture preservation measure. These local measures are summarized below.

Contrast to Noise Ratio (CNR) - measures the contrast between a feature of interest and background noise. In the $m$-th region of interest, CNR is defined as [44]

$$
C N R=10 \log \frac{\mu_{m}-\mu_{b}}{\sqrt{\sigma_{m}^{2}+\sigma_{b}^{2}}}
$$

where $\mu_{m}$ and $\sigma_{m}^{2}$ denote mean value and variance of the $m$-th ROI, respectively, and $\mu_{b}$ and $\sigma_{b}^{2}$ denote mean value and variance of the background region, respectively. From the definition of CNRm in (17) it is clear that this performance measure will be large if $m$-th ROI contains a prominent feature with larger mean $\mu_{m}$ than the background $\mu_{b}$, but with a rather small variance $\sigma_{m}^{2}$. For highly textured region (large $\sigma_{m}^{2}$ ) the evaluated contrast can mistakenly appear small. Therefore, we introduce a new performance measure (that we call texture preservation) to account for this important property of filters. 
Texture Preservation (TP) - we define a new measure of preserving texture in a region of interest as

$$
T P_{m}=\frac{\sigma_{m}^{2}}{\left(\sigma_{m}^{\prime}\right)^{2}} \sqrt{\frac{\mu_{d e n}}{\mu_{i n}}}
$$

where $\left(\sigma_{m}^{\prime}\right)^{2}$ is the standard deviation of the $m$-th ROI in the unprocessed input image (before noise suppression), $\mu_{d e n}$ is the mean value of the denoised image and $\mu_{i n}$ is the mean value of the noisy image. Ideally, $\mu_{d e n}$ should be equal to $\mu_{i n}$, but for some methods it does not hold. The role of the second term in (18) is to compensate for this change of the total mean and to evaluate the preservation of the texture regardless of the brightness level in the image. We average the $T P$ measure over the ROI's:

$$
T P=\frac{1}{M} \sum_{m=1}^{M} T P_{m}
$$

$T P$ ranges between 0 and 1 , and remains close to 0 for filters that severely flatten the image structures.

Edge Preservation (EP) - we start from a performance measure of [85] that correlates locally edges in the processed image and in the ideal noise-free image. Since in our case, the ideal image is not available, we correlate the edges in the processed and in the original noisy image, similarly like in [44], but locally over the selected ROI's rather than globally over the whole image. Our edge preservation measure for the $m$-th ROI is

$$
E P_{m}=\frac{\Gamma\left(\Delta I_{m}^{\prime}-\overline{\Delta I_{m}^{\prime}}, \Delta I_{m}-\overline{\Delta I_{m}}\right)}{\left.\sqrt{\Gamma\left(\Delta I_{m}^{\prime}-\overline{\Delta I^{\prime}{ }_{m}}, \Delta I_{m}^{\prime}-\overline{\Delta I^{\prime}}{ }_{m}\right.}\right) \Gamma\left(\Delta I_{m}-\overline{\Delta I_{m}}, \Delta I_{m}-\overline{\Delta I_{m}}\right)}
$$

where $I_{m}$ and $I_{m}^{\prime}$ are sub-matrices that contain the denoised and the input noisy image regions, respectively, in the $m$-th ROI. The operator $\Delta$ is a Laplacian operator. In practice, $\Delta I$ is a highpass version of $I$ obtained by convolving $I$ with a standard $3 \times 3$ approximation of the Laplacian operator. $\bar{I}$ denotes the empirical mean of $I$. $\Gamma$ operator denotes correlation inside the ROI:

$$
\Gamma\left(I_{1}, I_{2}\right)=\sum_{(i, j) \in R O I} I_{1}(i, j) I_{2}(i, j)
$$

We average the edge preservation measure over the $M$ selected ROI's:

$$
E P=\frac{1}{M} \sum_{m=1}^{M} E P_{m}
$$

This $E P$ measure ranges between 0 and 1 , having smaller values when the edges inside the ROI are more blurred.

Equivalent number of looks (ENL) - a commonly used performance measure for speckle suppression, which measures smoothness in areas that should appear homogeneous, but are corrupted by speckle. While in [44] this measure is averaged over the ROI's of an OCT image, we calculate it only in the background region. The reason for this is that only in the background we can safely assume that the ideal intensity should be homogeneous. Hence we evaluate

$$
E N L=\frac{\mu_{b}^{2}}{\sigma_{b}^{2}}
$$

where $\mu_{b}^{2}$ and $\sigma_{b}^{2}$ denote mean value and variance of the background region, respectively. A larger ENL indicates a stronger speckle smoothing in the corresponding region.

Table 2 shows the results of objective performance evaluation of the tested filters, with the performance measures described above, for three test images (the image from Fig. 9 and two other images from Fig. 10). A relative comparison between different filters (to be discussed in more detail in the next Section) is consistent over these different images. The average results over a set of 12 test images from Fig. 10 are shown in Table 3. From the results in Table 2 and Table 3, it is clear that all the tested filters improve (to a different extent) CNR, ENL and SNR in comparison to the original image. It is also clear that all the filters deteriorate edge preservation (EP) and texture preservation (TP) measures comparing to the original, since these are the requirements that have to be compromised against noise reduction.

\section{Software phantom evaluation}

We also perform an objective performance evaluation on our originally generated software phantom image. This software phantom image combines an artificially generated software phantom (Fig. 11(a)) and real noise extracted from a large uniform region of an actual OCT image as we explain next. Alternatively, we could have generated some simplified type of speckle noise artificially. However, there exist already many studies that compare different filters on artificially generated speckle noise and it is not our intention to repeat here such an analysis. We aim for OCT specific analysis with true noise characteristics.

In real OCT images that are at our disposal there is always a relatively large region with practically pure noise (see the bottom half of images in Fig. 4). We 
extract such a "pure noise region" of the same size as our noise-free phantom $\mathbf{f}$ and we form the noisy phantom $\mathbf{f}_{\mathbf{n}}$ by combining the noise-free phantom image $\mathbf{f}$ and the extracted noise image n. Since speckle is multiplicative noise, we will form $\mathbf{f}_{\mathbf{n}}$ by a point wise multiplication of $\mathbf{f}$ and $\mathbf{n}$, or equivalently, the logarithm of $\mathbf{f}_{\mathbf{n}}$ will be produced by summing $\log (\mathbf{f})$ and $\log (\mathbf{n})$. Note however that in reality mean value of the noise field $\mathbf{n}$ equals 1 . Since our noise field $\mathrm{n}$ is extracted not from raw OCT data but from an image in a classical image format, the mean value of $\mathbf{n}$ is not 1 but much larger. We have to compensate for this bias in the noise field and hence we generate the noisy image as:

$$
\log \left(\mathbf{f}_{\mathbf{n}}\right)=\log (\mathbf{f})+\log (\mathbf{n})-\log \left(\mu_{n}\right)
$$

Where $\mu_{n}$ denotes the mean value of the extracted noise field $\mathbf{n}$.

The resulting noisy image $\mathbf{f}_{\mathbf{n}}$ is shown in Fig. 11. This image has nearly the same noise statistics as real OCT images in Fig. 4 and Fig. 10, but now we have a ground truth for the signal structures.

The results of applying different filters to the noisy phantom image $\mathbf{f}_{\mathbf{n}}$ are shown in Fig. 12 and the PSNR values, for each denoised image $\mathbf{f}_{\text {den }}$, are calculated as

$$
P S N R=10 \log \frac{255^{2}}{\operatorname{Var}\left(\mathbf{f}-\mathbf{f}_{\mathbf{d e n}}\right)}
$$

To avoid the influence of the global intensity change produced by some methods (like RKT), we corrected the mean values of the images before calculating the PSNR in (25). The results are tabulated in Table 4 and visual results are shown in Fig. 12. These results on the phantom image show a similar comparison between the analyzed techniques as the evaluation on real OCT images in Section V.B. Single-resolution filters obviously produce excessive blurring. The results of the BLS-GSM and the WGE filters are better visually and in terms of PSNR than for the other filters. The WGE filter preserves best small details (see Fig. 12) and yields also the highest PSNR (see Table 4).

\section{DISCUSSION}

The quantitative evaluation results from Table 2 and Table 3 agree well with visual evaluation in Fig. 5 and Fig. 6. These results also indicate that different filters may be preferred depending on whether priority is given to detail and texture preservation or to stronger noise smoothing and more pronounced contrast of the most prominent features.

We tested two single-resolution filters (the Lee filter and RKT) and four multiresolution filters (OCTWT, FBT, WGE and BLS-GSM). The RKT method performs in this study better than the Lee filter, but both of these singleresolution filters appear to be inferior with respect to the multiresolution filters.

Out of all the tested filters, the feature based wavelet thresholding method FBT performs best in terms of ENL and SNR and scores also high in terms of CNR. This filter however gives worse results in terms of texture preservation (TP) and edge preservation (EP) measures, which is also evident from visual results in Fig. 5 and Fig. 6. This filter probably can be interesting as a preprocessing for segmentation of the most prominent features in the image but less interesting for visual enhancement of the image content because some important structures/details may get smoothened out. Exactly the opposite performance appears in the case of the WGE method with a small constant $\gamma$. In this case, the textural details and edges are best preserved but uniform areas are also less well flattened and hence ENL and SNR are only mildly improved comparing to the original. With increasing the constant, noise is stronger smoothed out, which also imposes increase in SNR, ENL and CNR measures, while EP and TP measures inevitably deteriorate, but not severely. This is evident both from Table 2 and from Fig. 5 and Fig. 6. The WGE filter with $\gamma=1$ shows a similar performance as BLS-GSM according to objective measures in Table 2 and also according to visual results. Similar is also performance of OCTWT, which shows slightly weaker noise suppression ability then WGE and BLS-GSM, but also a good texture preservation. If some of the remaining noise is tolerable, and the emphasis is on texture and edge preservation, then WGE filter with $\gamma=0.4$ and OCTWT seem best choices among the evaluated ones. Similar conclusions follow from the evaluation on the software phantom in Section V.C.

In summary, the filters that showed best compromise between noise smoothing and detail preservation in this study are OCTWT, BLS-GSM and WGE with $\gamma$ between 0.4 and 1. Out of these, BLSGSM is of highest complexity but is also the only one that takes into account noise correlation. OCTWT and WGE methods appear very attractive in terms of detail preservation and also in terms of complexity.

\section{CONCLUSION}

We gave a review of wavelet based denoising methods for OCT together with a performance evaluation. Different objective performance measures were assessed and also a novel measure for texture preservation was defined. The results of the presented objective performance analysis agree well with visual assessment. The results indicate that different filters have different strong points and that the choice of the particular filter may depend on the particular use (e.g., enhancement for visual inspection or pre-processing for segmentation of 
most prominent features/regions). Several filters were identified which offer a remarkable image quality improvement with a good compromise between detail preservation and noise smoothing.

\section{REFERENCES}

[1] Huang D, Swanson E, Lin C, Schuman J, Stinson W. Chang W, Hee M, Flotte T, Gregory K, Puliafito C, Fujimoto J Optical coherence tomography. Science 1991; 254: $1178-81$.

[2] Schmitt J. Optical coherence tomography (OCT): A review. IEEE Journal of Selected Topics in Quantum Electronics 1999; 5: 1205-15.

[3] Dubois A, Vabre L, Boccara A, Beaurepaire E. Highresolution full-field optical coherence tomography with a Linnik microscope. Applied Optics 2002; 41: 805-12.

[4] Bouma B, Tearney G. Eds. Handbook of Optical Coherence Tomography, New York: Marcel Dekker, 2002.

[5] Fercher A, Drexler W, Hitzenberger C, Lasser T. Optical coherence tomography - principles and applications. Institute of Physics Publishing - Reports on progress in Physics 2003; 66: 239-303.

[6] Goodman J. Some fundamental properties of speckle. J Opt Soc Am 1976; 66: 1145-50.

[7] Gupta S, Chauhan R, and Sexana S. Wavelet-based statistical approach for speckle reduction in medical ultrasound images. Med Biol Eng Comput 2004; 42:189192.

[8] Achim A, Bezerianos A, and Tsakalides P. Novel bayesian multiscale method for speckle removal in medical ultrasound images. IEEE Transactions on Medical Imaging 2001; 20: 772-83.

[9] Achim A, Tsakalides P, and Bezerianos A. SAR image denoising via Bayesian wavelet shrinkage based on heavy tailed modeling. IEEE Transactions on Geoscience and Remote Sensing 2003; 41: 1773-84.

[10] Michailovich O, Tannenbaum A. Despeckling of medical ultrasound images. IEEE Transactions on ultrasonics, ferroelectrics and frequency control 2006; 53: 64-78.

[11] Abbott J, Thursone F. Acoustic speckle: theory and experimental analysis. Ultrason Imag 1979; 1: 303-24.

[12] Wagner R, Smith S, Sandrik J, and Lopez H. Statistics of speckle in ultrasound B-scans. IEEE Transactions on Sonics and Ultrasonics 1983; 30: 156-63.

[13] Bashkansky M, Reintjes J. Statistics and reduction of speckle in optical coherence tomography. Optics Letters 2000; 25: 545-7.

[14] Karamata B, Hassler K, Laubscher M, Lasser T. Speckle statistics in optical coherence tomography. J Opt Soc Am 2005; 22:593-596.

[15] Pircher M, Goetzinger E, Leitgeb R, Fercher A, Hitzenberger C. Speckle reduction in optical coherence tomography by frequency compounding. J Biomed Opt 2003; 8:565-569.

[16] Andersen P, Thrane L, Yura H, Tycho A, Jorgensen T, Frozs M. Advanced modelling of optical coherence tomography systems. Physics in medicine and biology 2004; 49:1307-1327.

[17] Thrane L. Optical coherence tomography: modeling and applications. Ph.D. dissertation 2001. Riso National Laboratory, Roskilde, Denmark.

[18] Kholodnykh A, Petrova I, Motamedi M, Esenaliev R. Accurate measurement of total attenuation coefficient of thin tissue with optical coherence tomography. IEEE Journal on Selected Topics in Quantum Electronics 2003, 9: 210-21.

[19] Van Der Meer F, Faber D, Baraznji Sassoon D, Aalders M, Pasterkamp G, Van Leeuwen T. Localized measurement of optical attenuation coefficients of atherosclerotic plaque constituents by quantitative optical coherence tomography. IEEE Transactions on Medical Imaging 2005; 24: 1369-76.

[20] Hillman, T, Adie S, Seemann V, Armstrong J. Correlation of static speckle with sample properties in optical coherence tomography. Optics Letters 2006; 31: 190-2.

[21] Touzi R. A review of speckle filtering in the context of estimation theory. IEEE Transactions on Geoscience and Remote Sensing 2002; 40: 2392-404.

[22] Corsini G, Mossa A, Verrazzani L. Signal-to-noise ratio and autocorrelation function of the image intensity in coherent systems: sub-Rayleigh and super-Rayleigh conditions. IEEE Transactions on Image Processing 1996; 5: 132-41.

[23] Lankoande O, Hayat M, Santhanam B. Speckle modeling and reduction in synthetic aperture radar imagery. In Proceedings of ICIP05 2005; 317-20.

[24] Dainty J. Topic in applied physics: laser speckle and related phenomena. New York: Springer-Verlag, 1984.

[25] Lee J. Digital image enhancement and noise filtering by use of local statistics. IEEE Trans Pattern Analysis and Computer Intell 1980; 2: 165-68.

[26] Kuan D, Sawchuk A, Strand T, Chavel P. Adaptive noise smoothing filter for images with signal-dependent noise. IEEE Trans Pattern Analysis and Computer Intell 1985; 7: 165-77.

[27] Frost V, Stiles J, Shanmugan K, Holtzman J. A model for radar images and its applications to adaptive digital filtering of multiplicative noise. IEEE Trans Pattern Analysis and Computer Intell 1982; 4: 157-66.

[28] Verhoeven J, Thijssen J. Improvement of lesion detectability by speckle reduction filtering: a quantitative study. Ultrason Imag 1993; 15: 181-204.

[29] Rogowska J, Brezinski M. Evaluation of the adaptive speckle suppression filter for coronal optical coherence tomography imaging. IEEE Transactions on Medical Imaging 2000; 19: 1261-6.

[30] Rogowska J, Brezinski M. Image processing techniques for noise removal, enhancement and segmentation of cartilage OCT images. Physics in Medicine and Biology 2002; 47: 641-55.

[31] Yung K, Lee S, Schmitt J. Phase-domain processing of optical coherence tomography images. J Biomed Opt 1999; 4: 125-36. 
[32] Schmitt, J. Restoration of optical coherence tomography images of living tissue using the clean algorithm. J Biomed Opt 1998; 3:66-75.

[33] Hogbom J. Aperture synthesis with non-regular distribution of interferometer baselines. Astron Astrophys Suppl 1974; 15:417-426.

[34] Kulkarni M, Thomas C, Izatt, J. Image enhancement in optical coherence tomography using deconvolution. Electronic Letters 1997; 33: 1365-7.

[35] Marks D, Oldenburg A, Reynolds J, Boppart, S. Digital algorithm for dispersion correction in optical coherence tomography for homogeneous and stratified media. Appl. Opt 2003; 42: 204-17.

[36] Lee JS. Digital image enhancement and noise filtering by use of local statistics. IEEE Trans Pattern Anal Machine Intell 1980; 2: 165-8.

[37] Kuan DT, Sawchuk AA, Strand TC, Chavel P. Adaptive noise smoothing filter for images with signal dependent noise. IEEE Trans Pattern Anal Machine Intell 1985; 7:165-77.

[38] Lopes A, Touzi R, Nezry E. Adaptive speckle filters and scene heterogeneity. IEEE Transaction on Geosc Remote Sens 1990; 28:992-1000.

[39] Achim A, Bezerianos A, Tsakalides P. Novel Bayesian multiscale method for speckle removal in medical ultrasound images. IEEE Trans Medical Imaging 2001; 20:772-83, Aug. 001.

[40] Pižurica A, Philips W, Lemahieu I, Acheroy M. Despeckling SAR images using wavelets and a new class of adaptive shrinkage estimators. In Proc. IEEE Internat Conf Image Proc ICIP 2001, Thessaloniki, Greece.

[41] Pižurica A, Philips W, Lemahieu I and Acheroy M. A versatile wavelet domain noise filtration technique for medical imaging. IEEE Trans Medical Imaging 2003; 22: 323-31.

[42] Portilla J, Strela V, Wainwright MJ and Simoncelli EP. Image denoising using scale mixtures of Gaussians in the wavelet domain. IEEE Trans Image Proc. 2003; 12:133851.

[43] Xiang S, Zhou L and Schmitt J. Speckle noise reduction for optical coherence tomography. Proceedings SPIE (Euroser) 1997; 3196: 79-88.

[44] Adler D, Ko T and Fujimoto J. Speckle reduction in optical coherence tomography images by use of a spatially adaptive wavelet filter. Optics Letters 2004. 29, 2878-80.

[45] Ralston T, Marks D, Kamalabadi F and Boppart S. Deconvolution methods for migitation of transverse blurring in optical coherence tomography. IEEE Trans Image Proc 2005; 14:1254-64.

[46] Michailovich $O$ and Tannenbaum A. Despeckling of medical ultrasound images. IEEE Trans Ultrasonics, Ferroelectronics, and Frequency Control 2006; 53:64-78.

[47] Portilla J. Blind non-white noise removal in images using Gaussian scale mixtures in the wavelet domain. Proceedings of SPS 2004, 2004; 17-20.

[48] Xiang S and Zhang Y. Sensitivity enhancement using nonlinear optical wavelet thresholding for two- dimensional medical ultrasound transducer. Biomed Eng Appl Basis Commun 1997; 9:91-100.

[49] Ralston T, Atkinson I, Kamalabadi F and Boppart S. Multi-dimensional denoising of real-time OCT imaging data. Proceedings of ICASSP 2006; 1148.

[50] Mallat S. A theory for multiresolution signal decomposition: the wavelet representation. IEEE Trans Pattern Anal Machine Intell 1989; 11:674-93.

[51] Coifman RR and Donoho DL. In: Wavelets and Statistics, Antoniadis A and Oppenheim G Ed, Translation-invariant denoising. New York, Springer Verlag. 1995; 125-50.

[52] Chang SG, Yu B and Vetterli M. Spatially adaptive wavelet thresholding with context modeling for image denoising. IEEE Trans Image Proc 2000; 9:1522-31.

[53] Mallat S. A wavelet tour of signal processing. Academic Press, London, 1998.

[54] Donoho DL. De-noising by soft-thresholding. IEEE Trans Inform Theory 1995; 41:613-27.

[55] Donoho DL and Johnstone IM. Adapting to unknown smoothness via wavelet shrinkage. J Amer Stat Assoc 1995; 90:1200-24

[56] Cai T. Adaptive wavelet estimation: a block thresholding and oracle inequality approach. The Annals of Statistics 1999; 27: 898-924.

[57] Cai $\mathrm{T}$ and Silverman B. Incorporating information on neighboring coefficients into wavelet estimation. Sankhya 2001; 63: 127-48.

[58] Vidakovic B. Wavelet-based nonparametric Bayes methods. Practical Nonparametric and Semiparametric Bayesian Statistics, ser. Lecture Notes in Statistics 1998.

[59] Abramovich F, Sapatinas T and Silverman B. Wavelet thresholding via a Bayesian approach. J Royal Statist Society B 1998; 60: 725-49.

[60] Leporini D, Pasquet JC and Krim H. In: Müller P and Vidakovic B Ed, Best basis representation with prior statistical models. New York, Springer Verlag. 1999; 15572.

[61] Chipman HA, Kolaczyk ED and McCulloch RE. Adaptive Bayesian wavelet shrinkage. J Amer Statist Assoc 1997; 92: 1413-21.

[62] Clyde M, Parmigiani G and Vidakovic B. Multiple shrinkage and subset selection in wavelets. Biometrika 1998; 85: 391-401.

[63] Simoncelli EP, Adelson EH. Noise removal via Bayesian wavelet coring. Proc. IEEE Internat. Conf. Image Proc. ICIP, Lausanne, Switzerland, 1996.

[64] Hansen M, Yu B. Wavelet thresholding via MDL for natural images. IEEE Trans Inform Theory 2000; 46: 1778-88.

[65] Achim A, Tsakalides P, Bezerianos A. SAR image denoising via Bayesian wavelet shrinkage based on heavytailed modeling. IEEE Trans. Geosc and Remote Sens 2003; 41: 1773-84.

[66] Moulin P, Liu J. Analysis of multiresolution image denoising schemes using generalized Gaussian and complexity priors. IEEE Trans Inform Theory 1999; 45: 909-19. 
[67] Şendur L, Selesnick IW. Bivariate shrinkage with local variance estimation. IEEE Signal Proc Lett 2002; 9: 43841.

[68] Romberg JK, Choi H, Baraniuk RG Bayesian tree structured image modeling using wavelet-domain hidden Markov models. Proc. SPIE Technical Conf on Mathematical Modeling, Bayesian Estimation, and Inverse Problems, Denver, CO. 1999; 31.

[69] Fan G, Xia XG. Image denoising using local contextual hidden Markov model in the wavelet domain. IEEE Signal Proc Lett 2001; 8: 125-8.

[70] Mihçak MK, Kozintsev I, Ramchandran K, Moulin P. Low-complexity image denoising based on statistical modeling of wavelet coefficients. IEEE Signal Proc Lett 1999; 6: 300-3.

[71] Strela V, Portilla J, Simoncelli EP. Image denoising using a local Gaussian scale mixture model in the wavelet domain. Proc. SPIE 45th Annual Meeting 2000, San Diego.

[72] Portilla J, Strela V, Wainwright MJ, Simoncelli EP. Adaptive Wiener denoising using a Gaussian scale mixture model in the wavelet domain. Proc. IEEE Internat Conf on Image Proc, Thessaloniki, Greece 2001.

[73] Li X, Orchard M. Spatially adaptive denoising under overcomplete expansion. Proc. IEEE Internat Conf on Image Proc ICIP Vancouver, Canada 2000.

[74] Malfait M, Roose D. Wavelet-based image denoising using a Markov random field a priori model. IEEE Trans Image Proc 1997; 6: 549-65.

[75] Jansen M, Bultheel A. Empirical Bayes approach to improve wavelet thresholding for image noise reduction. J Amer Stat Assoc 2001; 96: 629-39.

[76] Pižurica A, Philips W, Lemahieu I, Acheroy M. A joint inter- and intrascale statistical model for wavelet based Bayesian image denoising. IEEE Trans Image Proc 2002; 11: 545-57.

[77] Pižurica A, Philips W. Estimating the probability of the presence of a signal of interest in multiresolution single and multiband image denoising. IEEE Trans Image Proc 2006;15: 654-65.
[78] Crouse MS, Nowak RD, Baranuik RG. Wavelet-based statistical signal processing using hidden Markov models. IEEE Trans Signal Proc 1998; 46: 886-902.

[79] Jansen M, Bultheel A. In: Bayesian inference in wavelet based models, ser. Lecture Notes in Statistics, Müller P and Vidakovic BB Ed, Geometrical priors for noise free wavelet coefficients in image denoising. New York, Springer Verlag. 1999; 223-42.

[80] Mallat S, Hwang WL. Singularity detection and processing with wavelets. IEEE Trans Inform Theory 1992; 38: 61743.

[81] Xu Y, Weaver J, Healy D, Lu J. Wavelet transform domain filters: a spatially selective noise filtration technique. IEEE Trans. Image Proc 1994; 3: 747-58.

[82] Marr D. In: Vision. New York, WH Freeman and Co. 1982.

[83] Balster E, Zheng Y, Ewing R. Feature-Based Wavelet Shrinkage Algorithm for Image Denoising. IEEE Trans Image Proc 2005; 14: 2024-39.

[84] Huysmans B, Pižurica A, Philips W. A geometrical wavelet shrinkage approach for image denoising 2006; European Signal Proc. Conf. EUSIPCO.

[85] Sattar F, Floreby L, Salomonsson G, Lovstrom B. Image enhancement based on a nonlinear multiscale method. IEEE Trans Image Proc 1997; 6: 888-95.

[86] Selesnick I, Baraniuk RG, Kingsbury NG. The Dual-Tree Complex Wavelet Transform 2005; IEEE Signal Proc Mag 2005; 22: 123-51.

[87] Starck JL, Candès EJ, Donoho DL, The Curvelet Transform for Image Denoising. IEEE Trans Image Proc 2002; 11: 670-84.

[88] Tessens L, Pižurica A, Alecu A, Munteanu A Philips W. Context adaptive image denoising based on joint image statistics in the curvelet domain, Journal of Electronic imaging 2008. (In Press).

[89] Guerrero-Colon JA, Manceray L, Portilla L. Image Restoration Using Space-Variant Gaussian Scale Mixtures in Overcomplete Pyramids. IEEE Trans Image Proc 2008; 17: $27-41$

[90] Jansen M. Noise Reduction by Wavelet Thresholding. New York, Springer-Verlag 2001. 

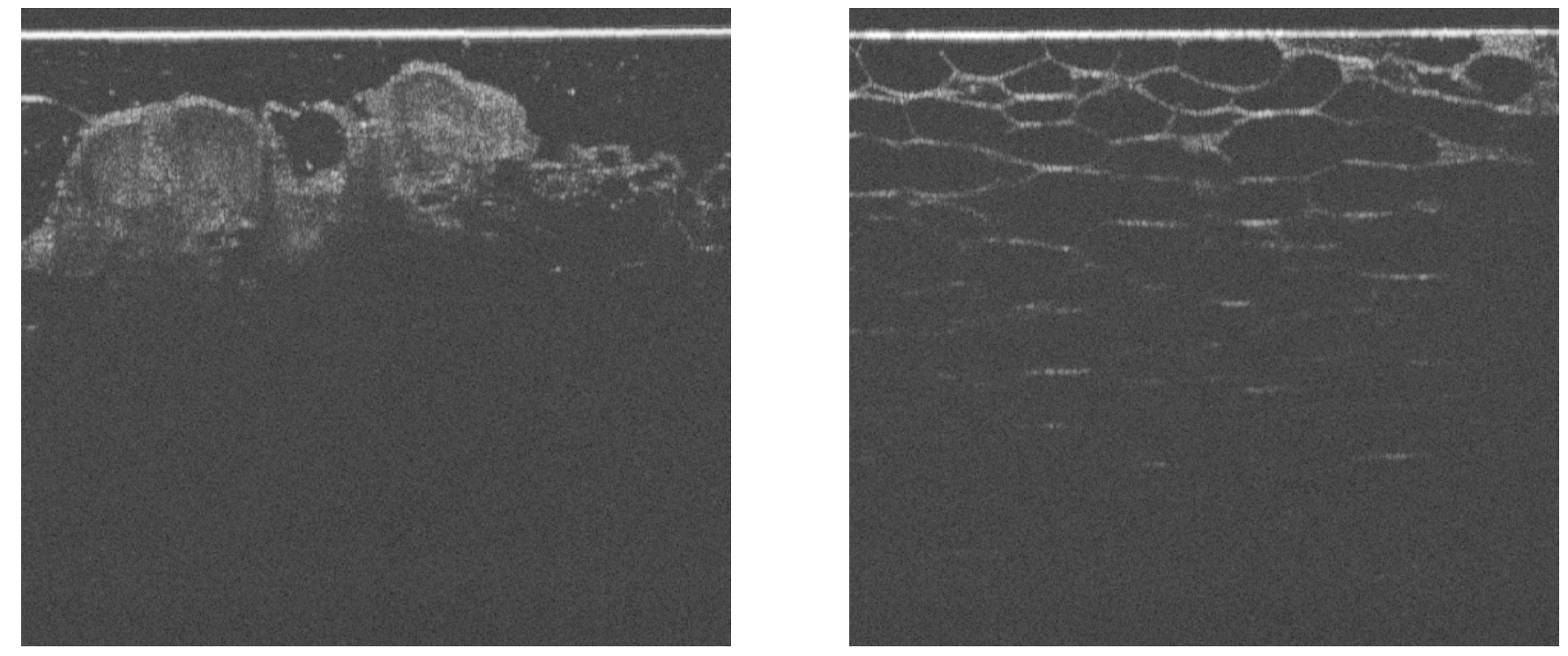

Fig.4. Examples of high-resolution OCT images used in our performance evaluation.

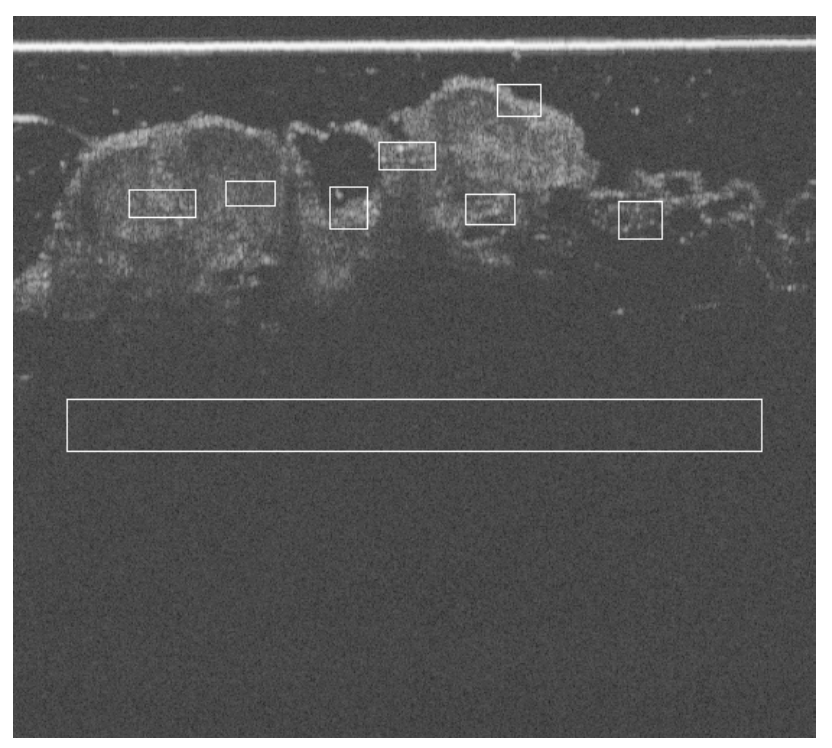

Fig. 9. Test image used in objective performance evaluation with chosen regions of interest. The seven rectangles in the upper part of the image are the ROI's and the large rectangle in the lower part is used as the background region. 

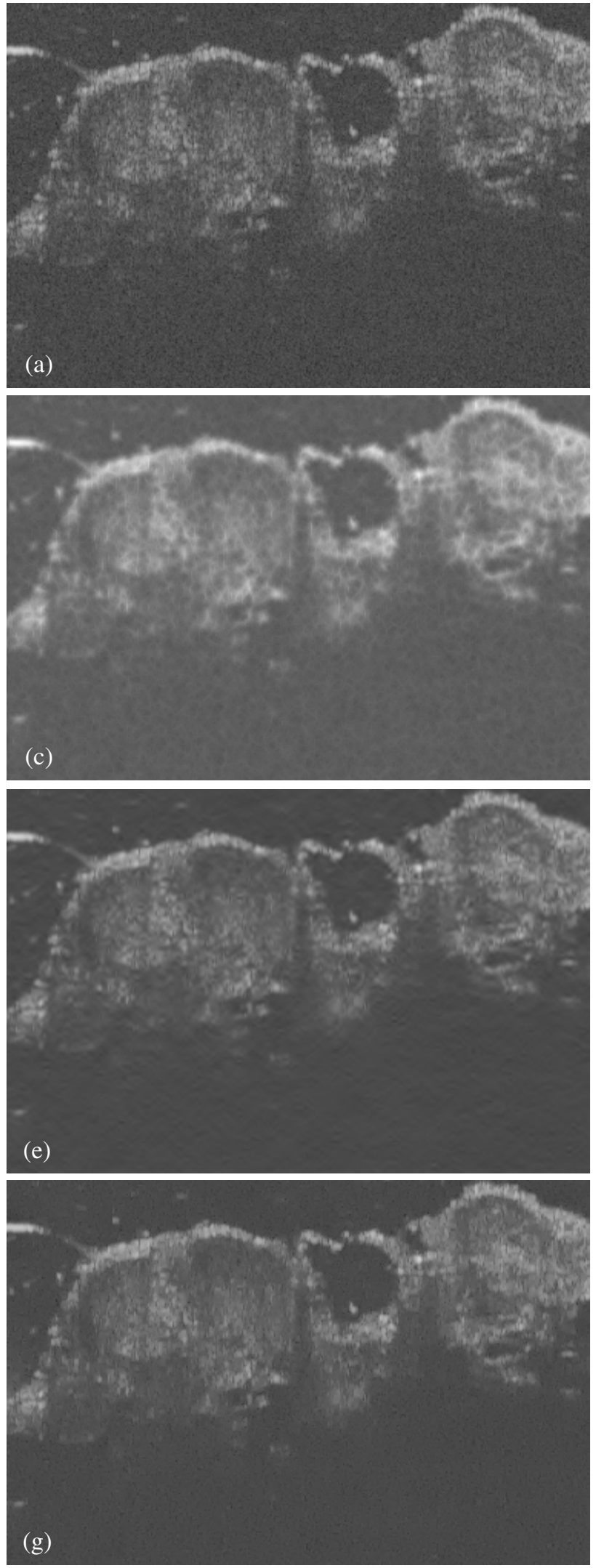

(b)
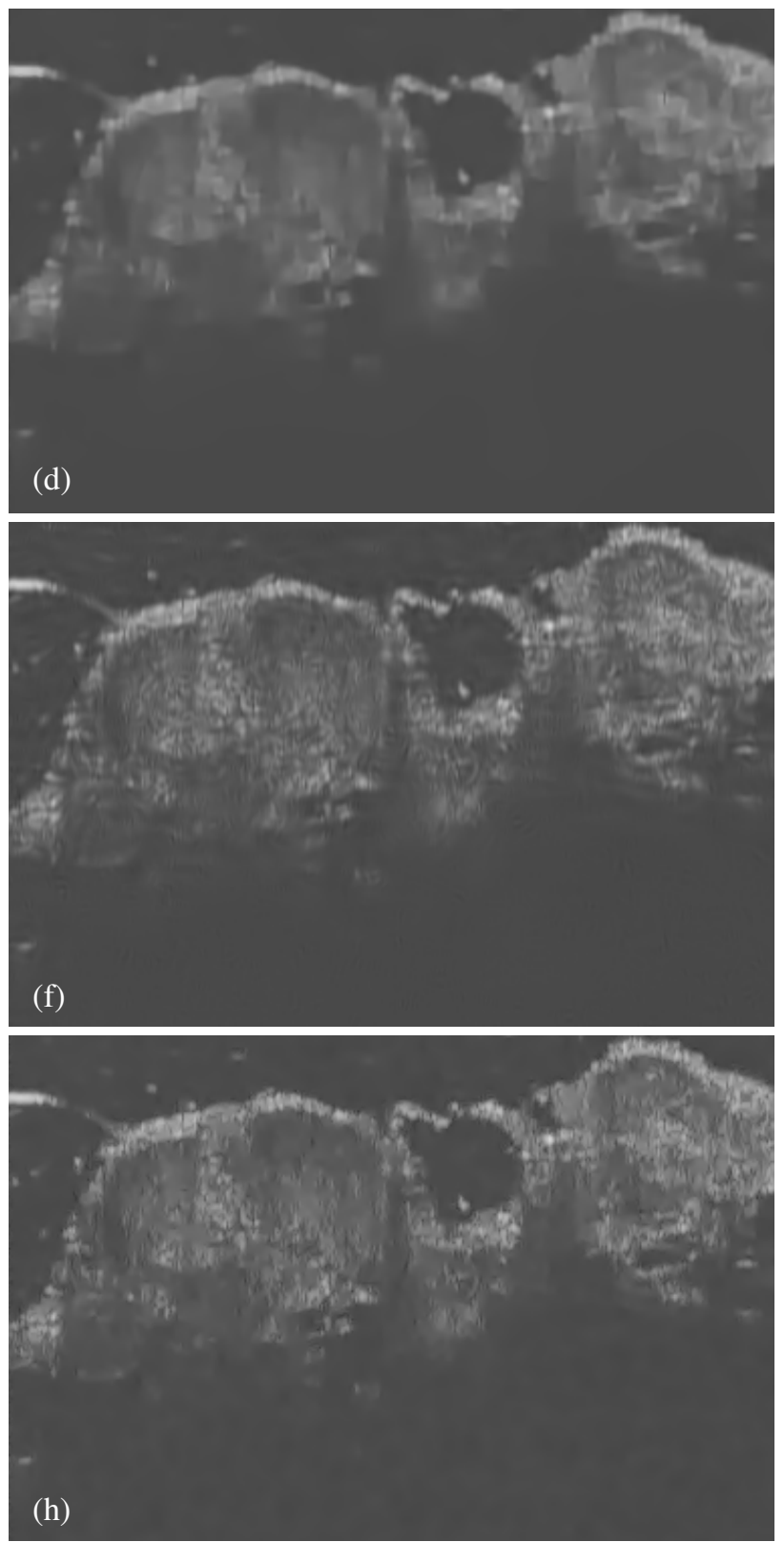

Fig. 5. (a) Noisy image (crop out) and the results of (b) Lee filter; (c) RKT filter [29,30]; (d) FBT [83]; (e) OCTWT [44]; (f) BLS-GSM 42], [47]; (g) WGE [40] with $\gamma=0.1$ and (h) WGE [40] with $\gamma=1$. 

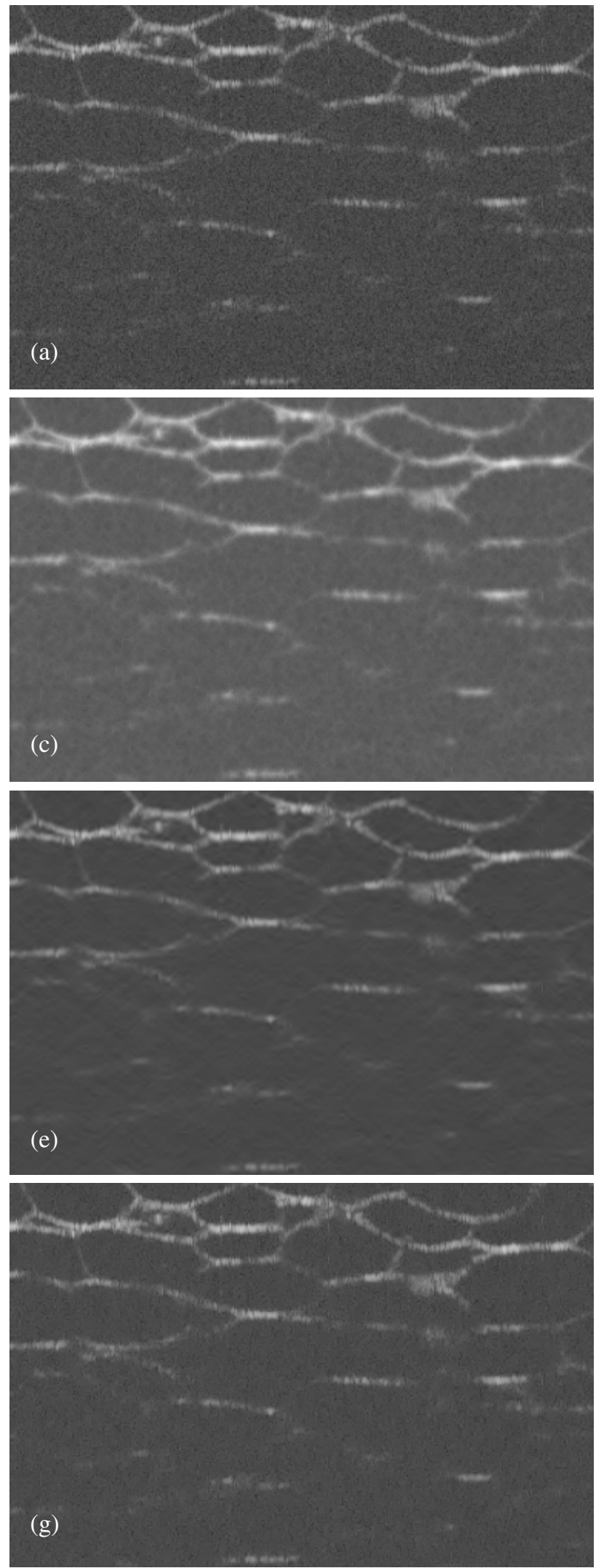

Fig. 6. (a) Noisy image (crop out) and the results of (b) Lee filter; (c) RKT filter [29,30]; (d) FBT [83]; (e) OCTWT [44]; (f) BLS-GSM 42], [47]; (g) WGE [40] with $\gamma=0.1$ and (h) WGE [40] with $\gamma=1$.
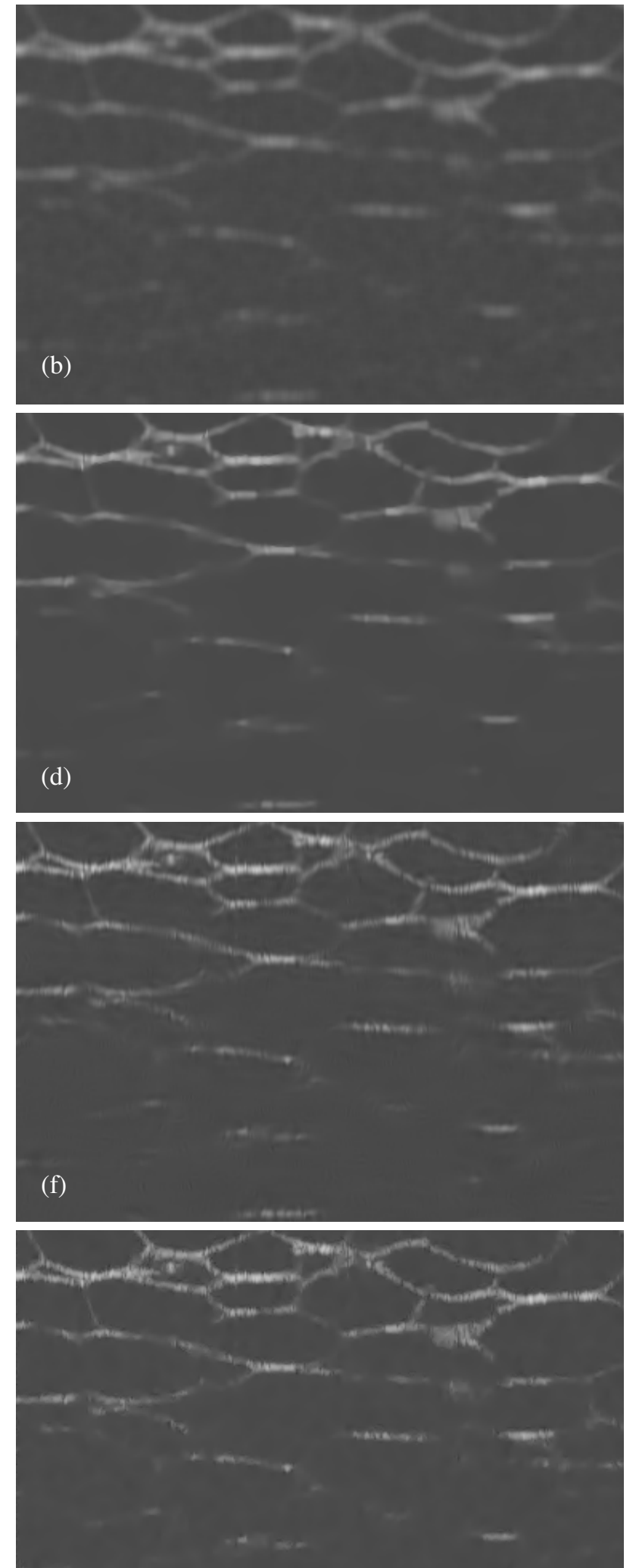

(h) 

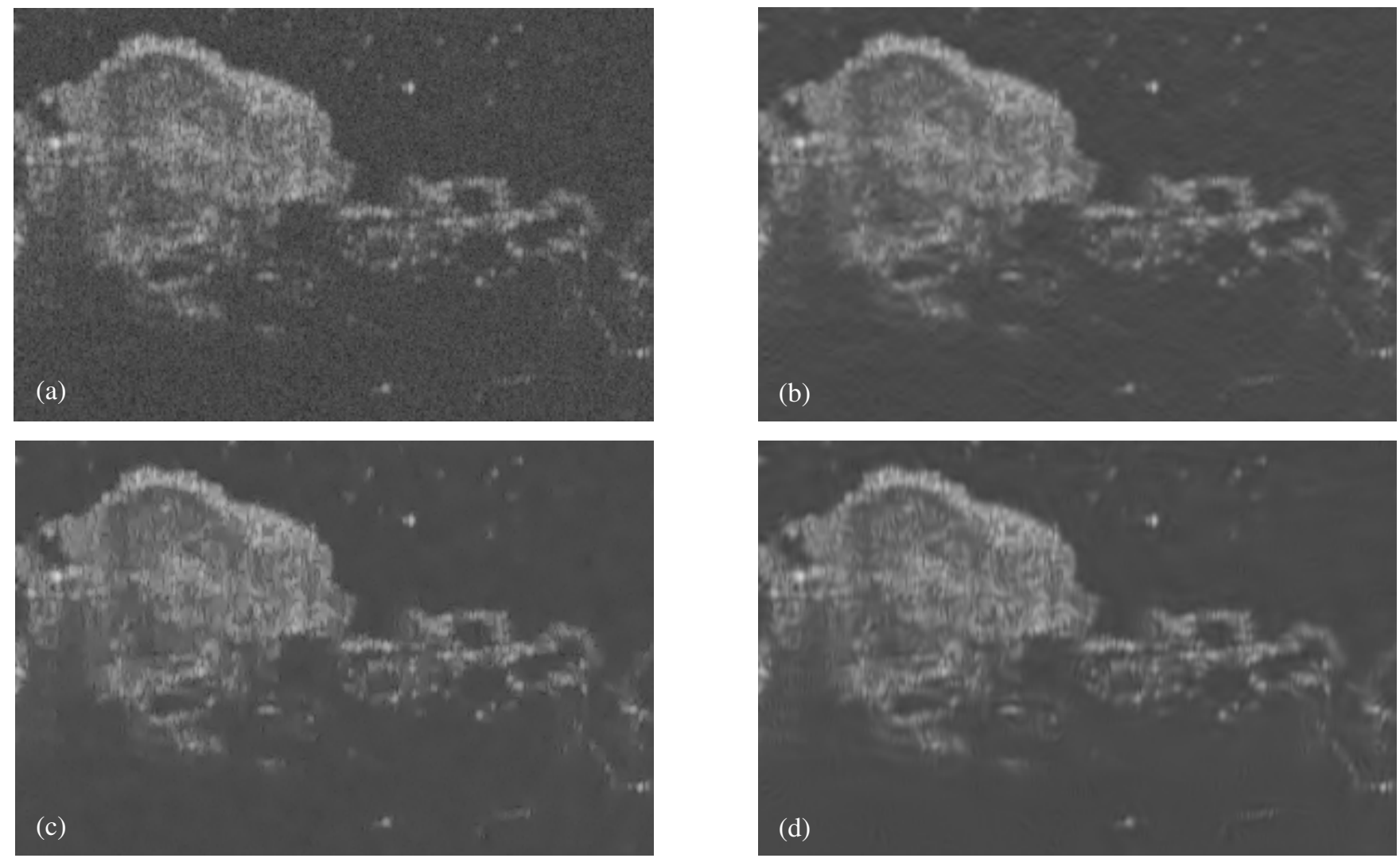

Fig. 7. An illustration of the results of the best performing filters in this study. (a) Noisy image detail and the results of (b) OCTWT [44]; (c) WGE [40] with $\gamma=0.4$ and (d) BLS-GSM 42], [47].
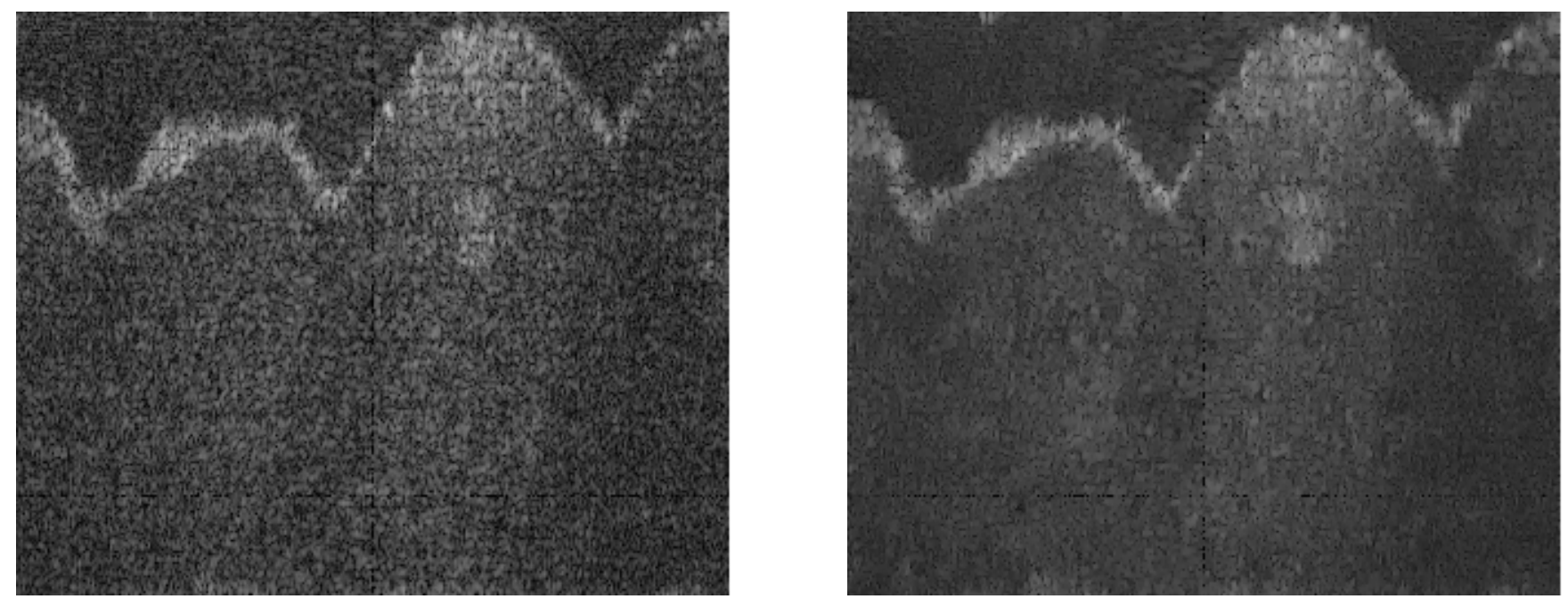

Fig. 8. (a) A part of an original OCT image and (b) the result of WGE [40] with $\gamma=0.4$. 
TEST IMAGE 1

\begin{tabular}{|l|r|r|r|l|l|}
\hline & CNR & ENL & SNR[dB] & TP & EP \\
\hline Original & 0.61 & 107.83 & 11.93 & 1.00 & 1.00 \\
\hline Lee & 2.30 & 457.87 & 23.21 & 0.78 & 0.52 \\
\hline RKT & 1.75 & 604.56 & 19.73 & 0.81 & 0.70 \\
\hline OCTWT & 0.98 & 892.22 & 19.80 & 0.86 & 0.89 \\
\hline FBT & 0.83 & 366.11 & 17.46 & 0.94 & 0.97 \\
\hline WGE $\gamma=0.2$ & 0.94 & 576.10 & 20.03 & 0.92 & 0.98 \\
\hline WGE $\gamma=0.4$ & 0.95 & 3189.43 & 26.24 & 0.91 & 0.95 \\
\hline WGE $\gamma=1$ & 0.93 & 4511.86 & 27.78 & 0.92 & 0.93 \\
\hline GSM-BLS & 1.03 & 2499.60 & 25.05 & 0.88 & 0.87 \\
\hline
\end{tabular}

TEST IMAGE 2

\begin{tabular}{|l|r|r|r|l|l|}
\hline & CNR & ENL & SNR[dB] & TP & EP \\
\hline Original & 0.27 & 105.79 & 13.94 & 1.00 & 1.00 \\
\hline Lee & 0.49 & 190.22 & 21.32 & 0.86 & 0.91 \\
\hline RKT & 0.65 & 7556.3 & 31.89 & 0.87 & 0.86 \\
\hline OCTWT & 0.39 & 350.16 & 19.01 & 0.97 & 0.98 \\
\hline FBT & 2.12 & 2842.1 & 24.57 & 0.45 & 0.60 \\
\hline WGE $\gamma=0.2$ & 0.45 & 676.2 & 21.75 & 0.95 & 0.98 \\
\hline WGE $\gamma=0.4$ & 0.44 & 3002.8 & 28.28 & 0.96 & 0.97 \\
\hline WGE $\gamma=1$ & 0.42 & 5042.9 & 30.58 & 0.97 & 0.95 \\
\hline GSM-BLS & 0.65 & 7556.3 & 31.89 & 0.87 & 0.86 \\
\hline
\end{tabular}

TEST IMAGE 3

\begin{tabular}{|l|r|r|r|l|l|}
\hline & CNR & ENL & SNR[dB] & TP & EP \\
\hline Original & 0.68 & 114.77 & 18.91 & 1.00 & 1.00 \\
\hline Lee & 0.19 & 244.79 & 24.48 & 0.73 & 0.92 \\
\hline RKT & 0.83 & 2985.1 & 32.71 & 0.92 & 0.92 \\
\hline OCTWT & 0.72 & 238.01 & 22.04 & 0.99 & 0.99 \\
\hline FBT & 1.86 & 2693.4 & 30.16 & 0.58 & 0.67 \\
\hline WGE $\gamma=0.2$ & 0.73 & 744.66 & 26.97 & 0.99 & 0.99 \\
\hline WGE $\gamma=0.4$ & 0.72 & 2986 & 33.03 & 0.99 & 0.98 \\
\hline WGE $\gamma=1$ & 0.71 & 4515.4 & 34.85 & 0.99 & 0.97 \\
\hline GSM-BLS & 0.83 & 2985.1 & 32.71 & 0.92 & 0.92 \\
\hline & & & & & \\
\hline
\end{tabular}

Table 2. Objective performance evaluation results for three test images. Test image 1: the image from Fig. 9; Test image 2: the image 8 from Figure 10 and Test image 3: the image 11 from Figure 10.

\begin{tabular}{|l|r|r|r|l|l|}
\hline & AVERAGE OVER A SET OF 12 TEST IMAGES \\
\hline & CNR & ENL & SNR[dB] & TP & EP \\
\hline Original & 0.91 & 108.54 & 14.63 & 1.00 & 1.00 \\
\hline Lee & 1.75 & 332.91 & 23.53 & 0.72 & 0.77 \\
\hline RKT & 2.04 & 607.68 & 22.10 & 0.80 & 0.74 \\
\hline OCTWT & 1.06 & 536.11 & 20.56 & 0.89 & 0.95 \\
\hline FBT & 1.93 & 1985.63 & 23.29 & 0.68 & 0.72 \\
\hline WGE $\gamma=0.2$ & 1.09 & 676.34 & 22.79 & 0.96 & 0.99 \\
\hline WGE $\gamma=0.4$ & 1.08 & 3296.44 & 29.24 & 0.96 & 0.97 \\
\hline WGE $\gamma=1$ & 1.05 & 5118.23 & 31.19 & 0.97 & 0.97 \\
\hline GSM-BLS & 1.22 & 5965.114 & 30.32 & 0.89 & 0.88 \\
\hline
\end{tabular}

Table 3. Objective performance evaluation results averaged over 12 test images from Figure 10.

\begin{tabular}{l} 
PSNR VALUES FOR PHANTOM IMAGE \\
\hline
\end{tabular}

Table 4. PSNR values for the reference methods. 


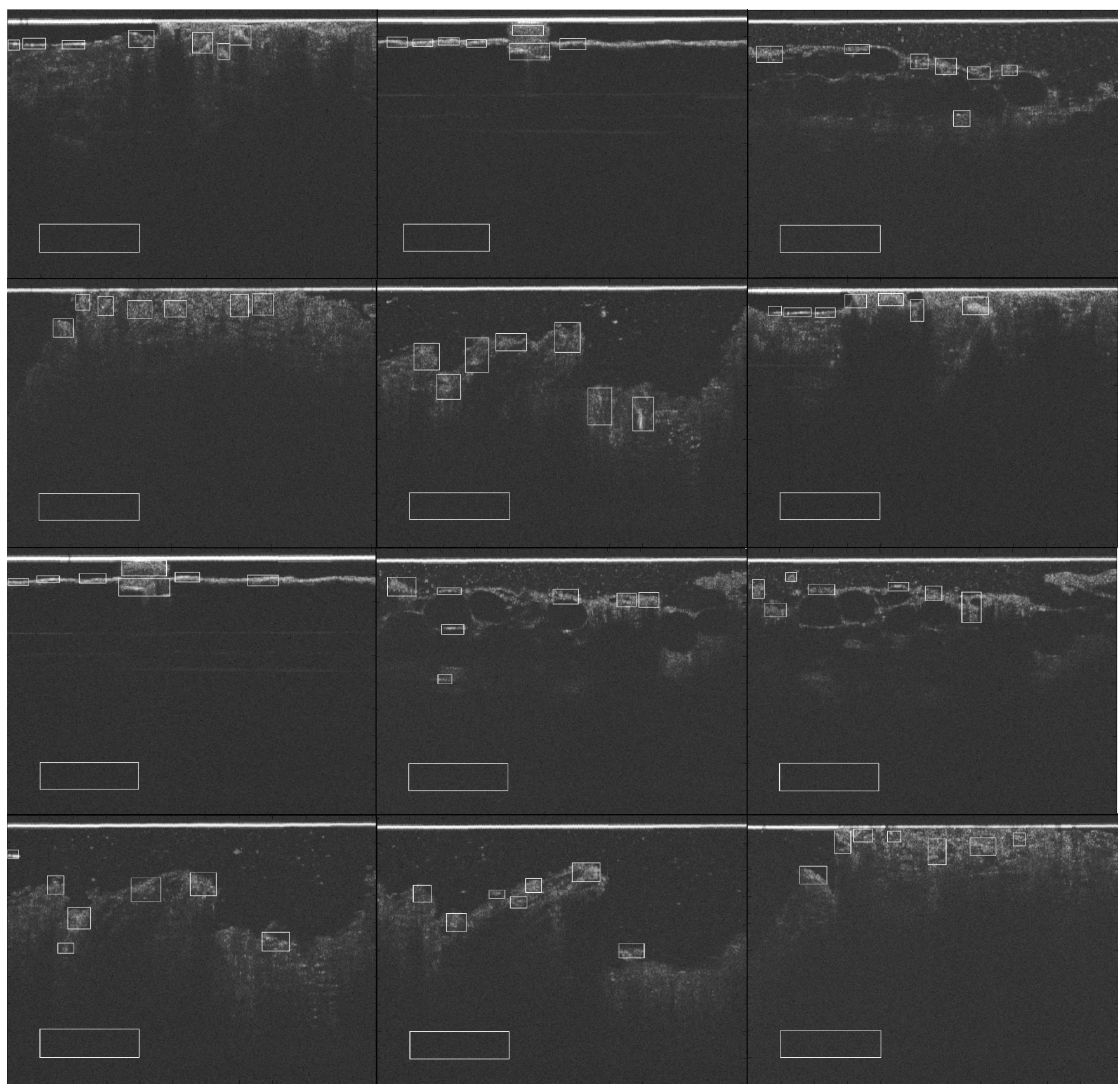

Fig. 10. A set of other 12 images (numbering left to right, top to bottom) used in objective performance evaluation together with ROI's, like in Fig. 9. 

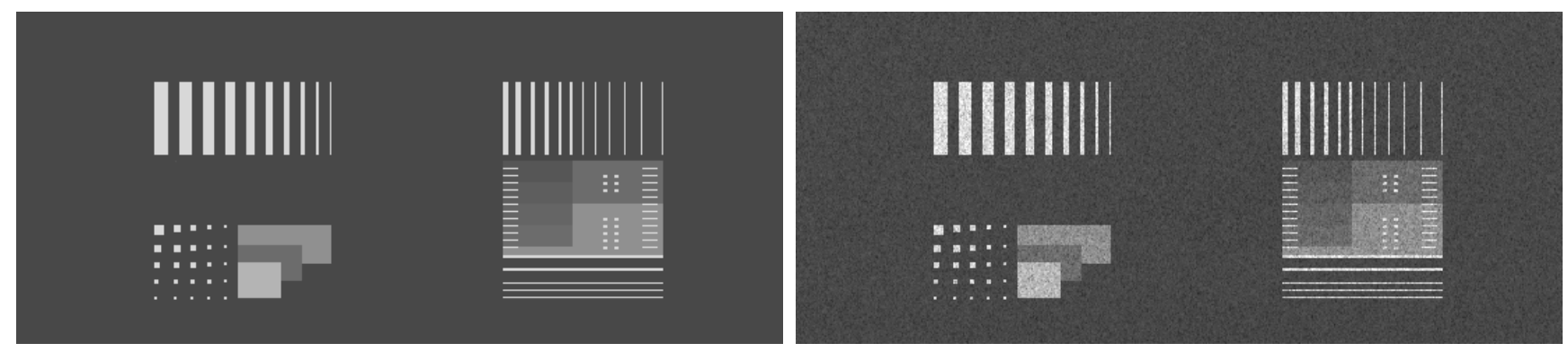

Fig. 11. The noise-free phantom image (left) and the noisy software phantom (right).
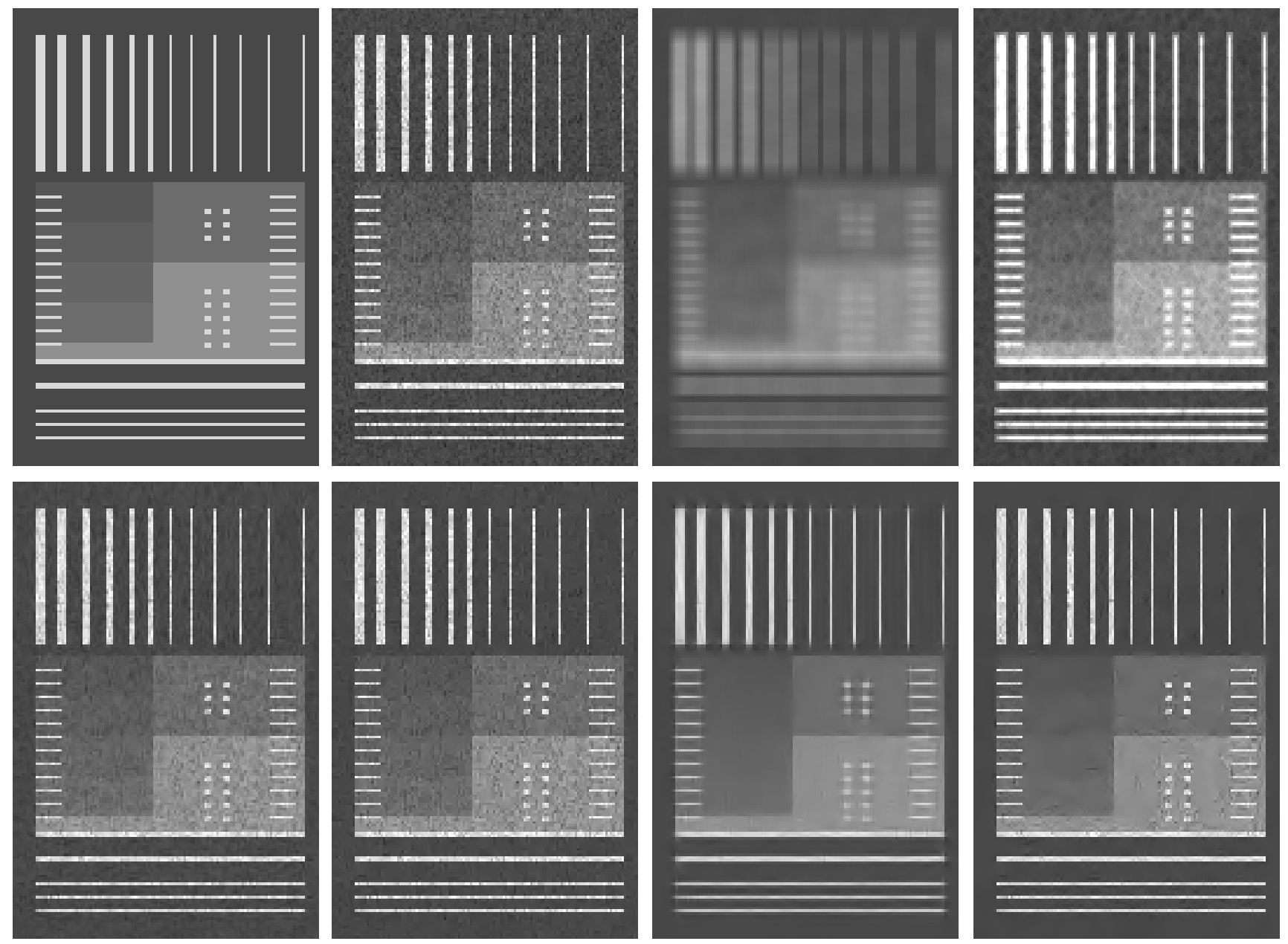

Fig. 12. A part of the phantom image with the corresponding results of the different analyzed method. First row, left to right: noise-free, noisy, the results of the Lee filter and the RKT filter. Second row, left to right: the results of the OCTWT, FBT, BLS-GSM and WGE filters. 